\title{
Automated Particle Shape Identification and Quantification for DEM Simulation of Rockfill Materials in Subgrade Construction
}

\author{
Hao Bai, ${ }^{1,2}$ Xiangyu Hu $\left(\mathbb{D},{ }^{3}\right.$ Ruidong Li $\mathbb{D}^{\circ},{ }^{3}$ Fei Chen, ${ }^{2,4}$ and Zhiyong Liao ${ }^{1,2}$ \\ ${ }^{1}$ Sichuan Expressway Construction \& Development Group Co.,LTD., Chengdu 610041, China \\ ${ }^{2}$ Shudao Investment Group Co.,Ltd., Chengdu 610041, China \\ ${ }^{3}$ Department of Civil and Environmental Engineering, The Hong Kong Polytechnic University, Hung Hom, Kowloon, Hong Kong, \\ China \\ ${ }^{4}$ Sichuan Intelligent High-Speed Technology Co.,Ltd, Chengdu 610000, China
}

Correspondence should be addressed to Xiangyu Hu; huxiangyu233@outlook.com

Received 13 June 2021; Revised 23 October 2021; Accepted 23 November 2021; Published 4 March 2022

Academic Editor: Qiao Dong

Copyright (C) 2022 Hao Bai et al. This is an open access article distributed under the Creative Commons Attribution License, which permits unrestricted use, distribution, and reproduction in any medium, provided the original work is properly cited.

\begin{abstract}
Rockfill materials, conducted by impermeable stone, are frequently used in subgrade construction projects. The irregularity and variability of particle shape are demonstrated to affect the mechanical properties of rockfill subgrade, such as void ratio and coordination number. This study first identifies the subgrade rockfill particle contour by machine learning algorithms, including AdaBoost, Cascade, and sliding windows. Then, the shape evaluation indexes of length flatness, edge angle, and roughness are quantified, and the statistical analysis of each index is presented. In addition, the discrete element method (DEM) simulation is implemented on the compaction of rockfill subgrade to explore the impact of roundness on characteristics of particles. Finally, the macroanalysis on the void ratio and cumulative settlement and the microanalysis on particle coordination number, rotation momentum, and displacement are studied. The results illustrate that roundness has a significant effect on the mechanical characteristics of subgrade rockfill materials. With the increase of rolling passes, the porosity of packing decreases, whereas the settlement increases gradually. The change rate starts fast and ends slowly.
\end{abstract}

\section{Introduction}

Rockfill materials have excellent engineering characteristics such as good compaction, strong water permeability, high filling density, high shear strength, small subsidence and deformation, high bearing capacity, and not easy to liquefy under the action of seismic load. Rockfill materials are applied in construction fields frequently for their excellent characteristics [1], meeting the requirements of in situ materials. In subgrade engineering, rockfill is mostly used, and it can make full use of local natural materials and adapt to different geological conditions.

The rockfill material method is relatively simple and possesses good seismic performance. Also, the evaluation of seismic reliability is an essential factor that needs to be considered. Zhang et al. evaluated geometric anisotropy modeling and shear behavior of graded crushed rocks [2].
There is research showing the seismic reliability of rockfill in hydraulic engineering under stochastic earthquake excitation considering the strain-softening behavior of rockfill materials. A new and efficient methodology that combines generalized probability density evolution method (GPDEM) with a spectral representation-random function method is presented to assess the seismic reliability [3].

To calculate the properties and state of rockfill materials more accurately, numerical simulation is a popular tool to make further research on rockfill materials [4]. For example, Li et al. researched by considering moisture and stress sensitivity of subgrade soils [5]. Hui et al. investigated the fracture evolution of asphalt mixture compared with acoustic emission [6]. The research found that particle breakage has some significant effects on the behavior of granular materials, and the shear strength and compressibility are also included. Raisianzadeh et al. [7] conducted a micromechanical study on particle 
breakage in 2D angular rockfill materials under biaxial compression loading using a combined DEM and extended finite element method (XFEM) approach. Song et al. [8] investigated the skeleton behaviors of open-graded friction courses using DEM. Zhou et al. [9] presented simulating tri-axial compression tests on numerical samples reveals the relationships among the micromechanical parameters and macroscopic response of rockfill materials.

In engineering, the identification and quantification of particles help to realize the needs of material classification and risk prediction, intelligent construction and management, and computer deep learning. To explore the effect of particle shape, Li et al. simulated three dimensions of aggregate and asphalt mixture using parameterized shape and size gradation [10]. And $\mathrm{Ma}$ et al. used another discrete method by a microscopic modeling approach to simplify the discrete element's shape by introducing irregular convex polyhedrons to reproduce the geometry-dependent behavior of rockfills [11]. In addition, in the research of Zhou et al., the deformable DEM was employed to study the effect of particle shape on the macroscopic mechanical behavior of rockfill materials [9], indicating that the particle shape has a significant impact on the macroscopic response of rockfill. On top of that, Nie et al. [12] explored the impacts of Fourier-based particle shape on the macro-mesoscopic shear responses of rockfill materials by numerical biaxial compression tests. However, these works on the impact of particle shape are limited, since most of them were conducted by numerical tests, which is time-consuming and expensive. Furthermore, the complete particle shape is not extracted and processed.

The previous methods have achieved the identification of particles to a certain extent, but they have not studied the effects of particle size and shape in depth. In this study, the rockfill particle contour is firstly extracted based on machine learning algorithms such as AdaBoost, Cascade, and sliding windows. Then, the shape evaluation indexes are quantified and the statistical analysis of each index is presented. In addition, the DEM simulation is implemented on the deformation and compaction of rockfill. Based on the simulation, the macroanalysis on void ratio and cumulative settlement is given. Finally, the microanalysis on particle coordination number, rotation momentum, and displacement is studied. Overall, this study lays a foundation for further research on the effect of particle shape and size of rockfill.

\section{Automated Shape Identification}

In this study, low-precision photography equipment (such as mobile phones and digital cameras) is used to obtain multiple subgrade rockfill materials images to save time and cost, considering the characteristics of the large number and low-precision requirements of rockfill material samples, as shown in Figure 1. The target of this study is to extract the irregular shape of subgrade rockfill materials.

Due to the simplicity, high precision, and high computational efficiency of the algorithm, the improved Viola-Jones algorithm [13] is used to automatically identify subgrade rockfill particles.
2.1. Training Set Preparation. Firstly, the most common shape of subgrade rockfill particles is considered. All the images containing a particle have an aspect ratio of $1: 1$. About 1206 pictures of these rockfill particles are taken, and then, some of these particles are cropped and zoomed as a training dataset for the model. Each cropped image is a small square in the original image. Among them, the images containing the complete outline of the subgrade rockfill particle are marked as positive images, and the other images are marked as negative images. All the cropped images have a size of $32 \times 32$ pixels. Taking these images as input data, the computer can train the model and learn to obtain Haar-Like features [14]. Then, the feature results are stored in the classifier. The sample images are shown in Figure 2.

2.2. Scaling and Rotation of Training Images. Mostly, the aspect ratio of subgrade rockfill particles is $1: 1$ to $2: 1$. Therefore, for particles beyond this range of ratio, it is difficult to collect a large amount of image data by photographing. However, there exist some real rockfill particles with a high aspect ratio, so the classifier is expected to detect these particles as well. Therefore, a new method of acquiring a large number of images is needed. In this study, to quickly obtain a large number of slender rockfill particle images that can be used for training, a stretching method is used to digitally stretch the existing images with an aspect ratio of 1 : 1 [10]. By adjusting the size of the previous $1: 1$ training image $(32 \times 32$ pixels $), 1.5: 1$ training image $(48 \times 32$ pixels), $2: 1$ training image $(64 \times 32$ pixels $), 3: 1$ training image $(96 \times 32$ pixels), and $4: 1$ training images $(128 \times 32$ pixels $)$ can be obtained.

The aspect ratio of most railway subgrade rockfill particles is $1: 1$ to $2: 1$. Therefore, for rockfill that is not in this ratio, it is difficult to collect a large amount of image data by shooting. However, these high aspect ratio rockfill particles are real, so the classifier also needs to detect this rockfill. Therefore, we need a new method of acquiring a large number of images. To quickly obtain a large number of slender rockfill particle images that can be used for training, this article digitally stretches the existing images with a rockfill aspect ratio of $1: 1$ [15] by adjusting the size of the previous $1: 1$ training image $(32 \times 32$ pixels $), 1.5: 1$ training image $(48 \times 32$ pixels), $2: 1$ training image $(64 \times 32$ pixels $), 3$ : 1 training image $(96 \times 32$ pixels) and $4: 1$ training images $(128 \times 32$ pixels), as shown in Figure 3 .

In addition, to recognize slender subgrade rockfill particles in any direction, the original image is rotated counterclockwise in $15^{\circ}$ increments, as shown in Figure 4 . For each rotated image, the sliding window scans the entire image in turn. The sliding window method may not be able to detect some subgrade rockfill particles due to the oblique placement of these particles. However, if the image is rotated, these particles will be converted to the vertical direction, which is easy to identify. After rotating the image by $15^{\circ}, 30^{\circ}, 45^{\circ}, 60^{\circ}, 75^{\circ}, 90^{\circ}, 105^{\circ}, 120^{\circ}, 135^{\circ}, 150^{\circ}$, and $165^{\circ}, 13$ rotated images can be obtained. Figure 4 shows a simple example of $0^{\circ}, 30^{\circ}$, and $60^{\circ}$. 


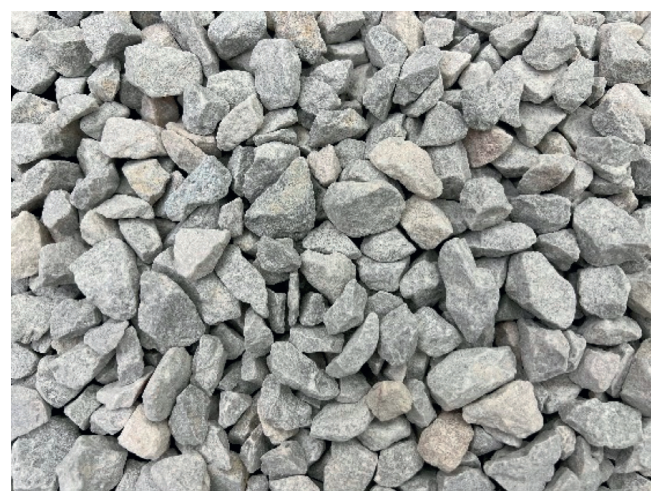

FIGURE 1: Schematic of subgrade rockfill particles.
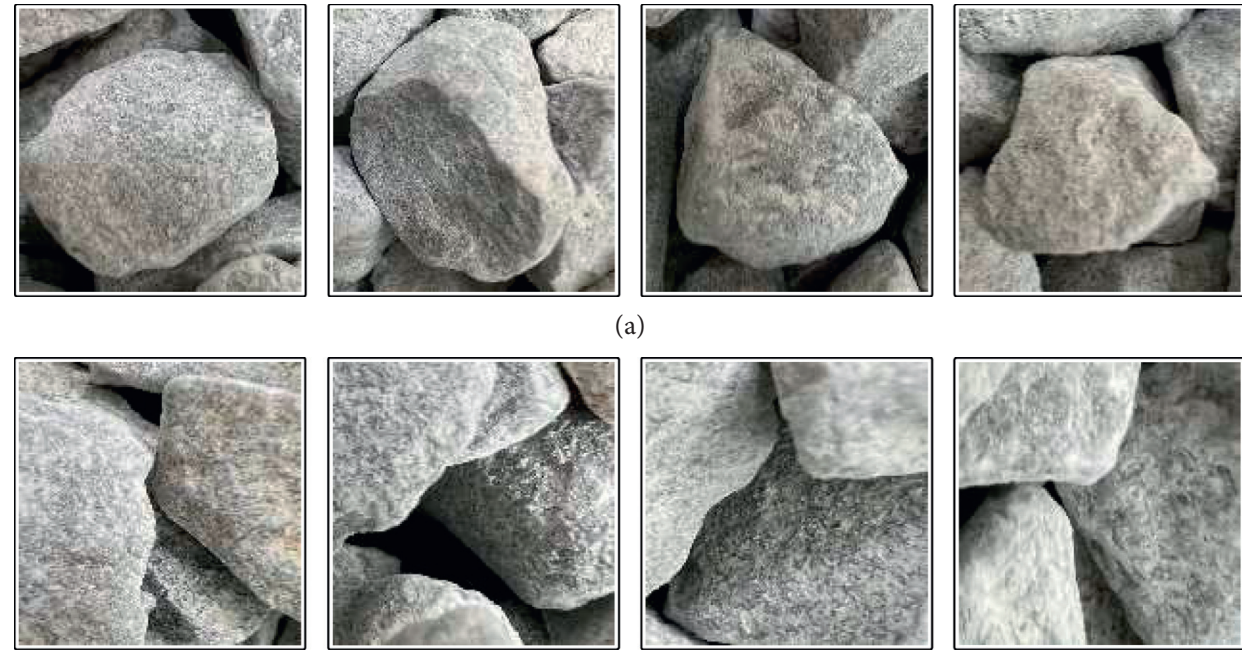

(b)

FIgURE 2: (a) Samples of positive images; (b) samples of negative images.

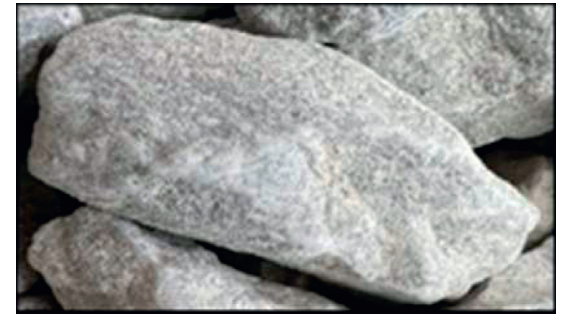

(a)

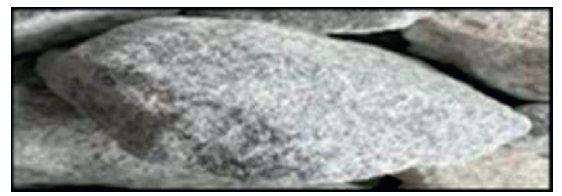

(c)

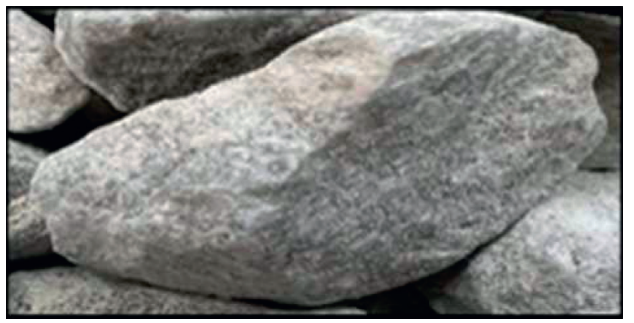

(b)

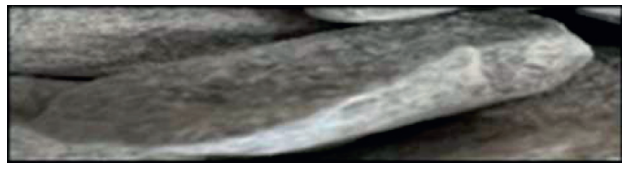

(d)

FIGURE 3: Schematic of stretching images: (a) 1.5:1 image; (b) 2:1 image; (c) $3: 1$ image; (d) $4: 1$ image.

Then, all the results of these images are merged. In each image, if a subgrade rockfill particle is successfully identified, a bounding box is added. Then, all bounding boxes will be superimposed when merging. However, due to the rotation, the same rockfill particle will generate some different bounding boxes. Thus, the image is rotated to the $0^{\circ}$ position, 


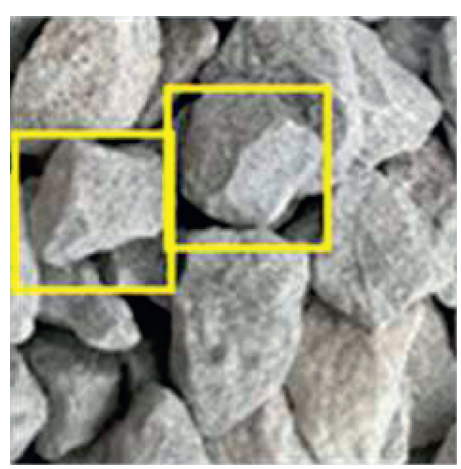

(a)

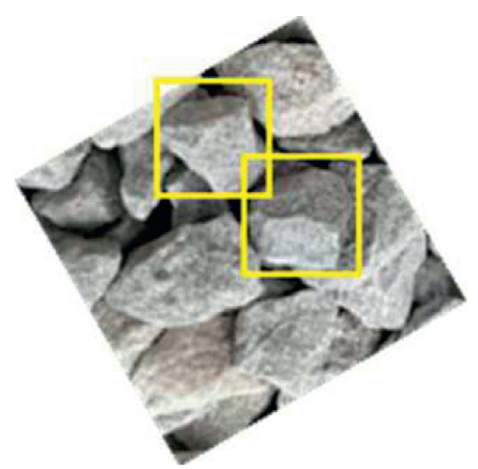

(b)

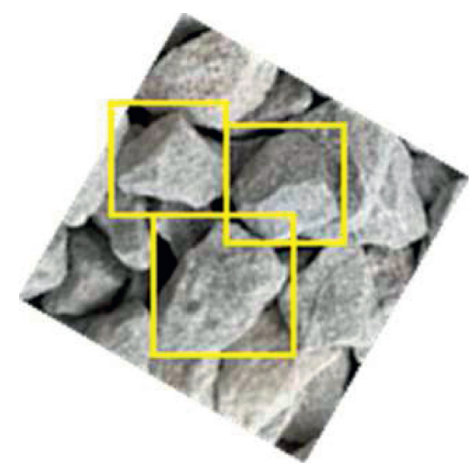

(c)

FIgURE 4: Schematic of rotation: (a) $0^{\circ}$; (b) $30^{\circ}$; (c) $60^{\circ}$.

which is equivalent to only rotating the sliding window. Then, the bounding box with the smallest area is selected as the final bounding box of this rockfill particle, as shown in Figure 5.

2.3. AdaBoost and Cascade Classifier. All the labeled images are used to train the model to obtain the feature difference between the positive and negative images in a dataset. Firstly, AdaBoost and Cascade methods [16] are used. Then, through the sliding window method [17], the subgrade rockfill particles can be successfully identified.

AdaBoost is a classification algorithm that can integrate some weak classifiers into a strong classifier. This strong classifier can be used in the Cascade method. The classifier is used to test an instance, and each classifier $f_{t}$ will output the probability distribution of two classes of positive or negative. These probabilities are combined in proportion to the relevant multipliers. The detailed algorithm is shown in Figure 6.

The training process of the Cascade method is divided into multiple stages. At each stage, a strong classifier is trained through the AdaBoost algorithm. In addition, three parameters need to be set in advance: the number of stages $s$, the detection rate $d$, and the false positive rate $f$, where $d$ represents the minimum percentage of correct detections and $f$ represents the maximum percentage of negative images that are incorrectly identified as positive. In each stage, it is necessary to search for the smallest $T$ in AdaBoost algorithm, so that the detection rate of the strong classifier $C(x)$ trained by AdaBoost is greater than $d$ and the false positive rate is less than $f$.

In the hypothetical example of Figure 7, 100 positive images and 100 negative images are used as input data. The original parameters of each stage are $s=3, d=0.99$, and $f=0.25$. Then, in each stage, a strong classifier with appropriate $T$ is trained through AdaBoost, with the value of $T$ 3,10 , and 35 , respectively. In the first stage, the model has 200 input images, and the classifier meets the requirements that the detection rate is greater than $d$ and the false positive rate is less than $f$. Then, the second stage is similarly performed. In stage 3 , the total detection rate $D=d^{3}=0.97$ and the false positive rate $F=f^{\beta}=0.015$ can be obtained.
2.4. Identifying Particles Based on the Sliding Window Method. Through the previously obtained classifier, images containing real particles can be obtained. The next task is to identify the complete contour of subgrade rockfill particles. Given a new image of rockfill particles, at each scanning position, the area in the sliding window is input to the detector using a sliding window algorithm. The detector will find and extract features in the window area of the image. Based on these features, the classifier finally determines whether the image is a positive image or a negative image. If it is a positive image, a bounding box is added to the scanning position to identify it as an area containing complete outline particles. The specific instructions are as follows.

Since the smallest pixel of the particle is about $50 \times 50$, a $50 \times 50$ sliding window is first used to scan the entire image. In each window, the crop image is input to the classifier. Since the previous classifiers are trained based on $32 \times 32$ images, cropped images larger than $32 \times 32$ should be scaled to $32 \times 32$, and the $C_{1}(x)$ classifier in the Cascade algorithm is tested. If the output is positive, move to stage 2 of the Cascade method. Otherwise, the negative images are rejected, and the sliding window will move to the next position by 1 pixel. If the cropped image successfully passes all $s$ classifiers, it will be a positive image containing the complete contour rockfill particles with a high probability. Then, the sliding window at this position is marked as the bounding box of the particle. Next, continue to move the sliding window until the entire image is scanned. The moving increment of the sliding window in the horizontal direction and the vertical direction is 1 pixel, and the moving path is shown in Figure 8(a).

When the $50 \times 50$ sliding window successfully scans the entire image, we add 5 pixels to the size of the sliding window in both horizontal and vertical directions, as shown in Figure 8(b). The previous sliding process is repeated until the sliding window expands to the maximum size of $300 \times 300$ pixels. Since most of the cropped images will be classified as negative images and rejected, only a small number of images can successfully pass all $s$ classifiers, so the algorithm has high computational efficiency.

Note that when the sliding window is large enough, several movements of the sliding window may contain the 


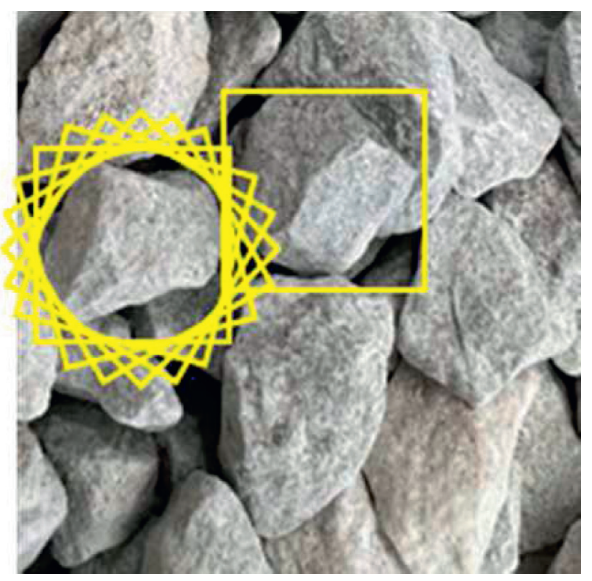

(a)

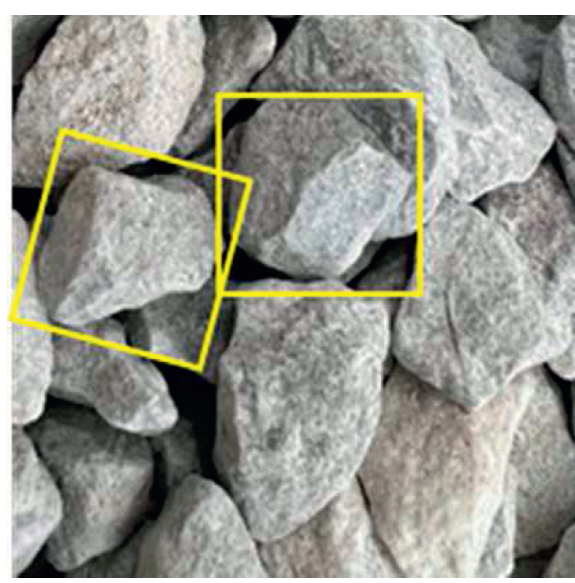

(b)

Figure 5: (a) The merges results of all rotation; (b) the average window of particles.

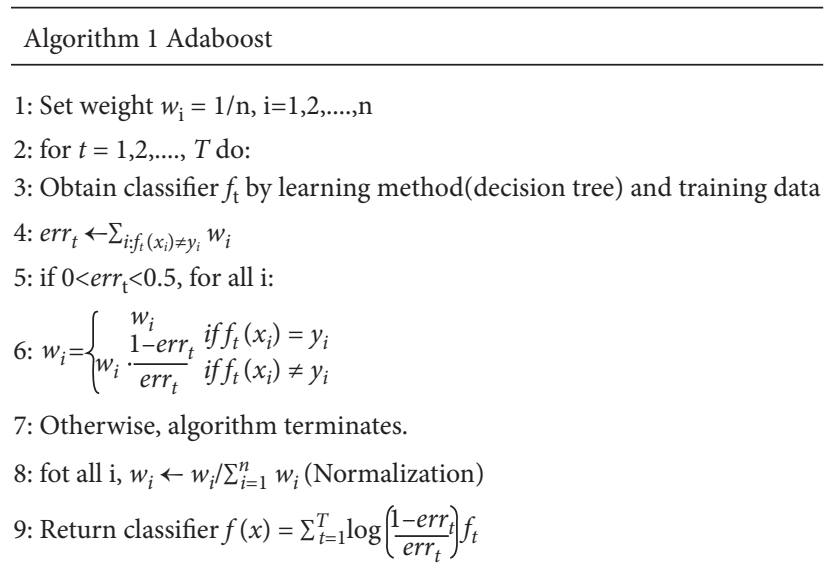

Figure 6: AdaBoost algorithm.

Stage 1

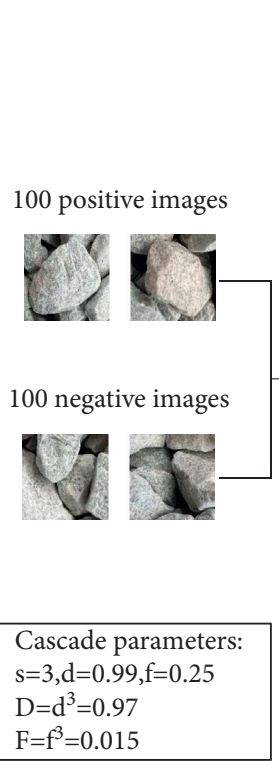

Stage 2

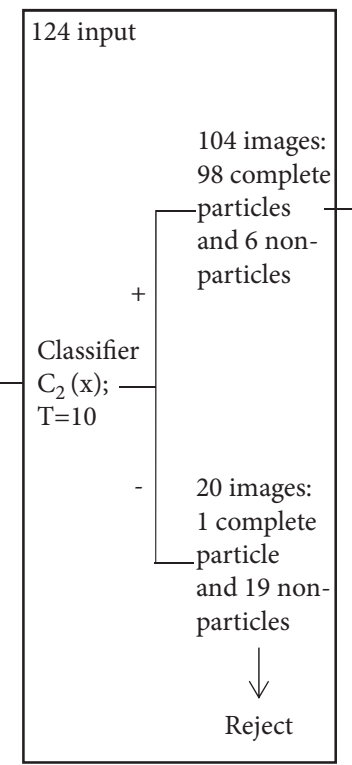

Stage 3

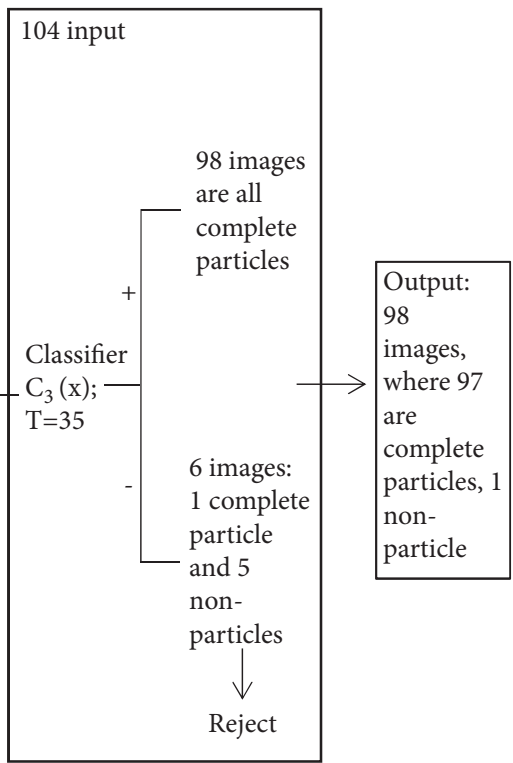

Figure 7: A hypothetical example of the Cascade algorithm. 


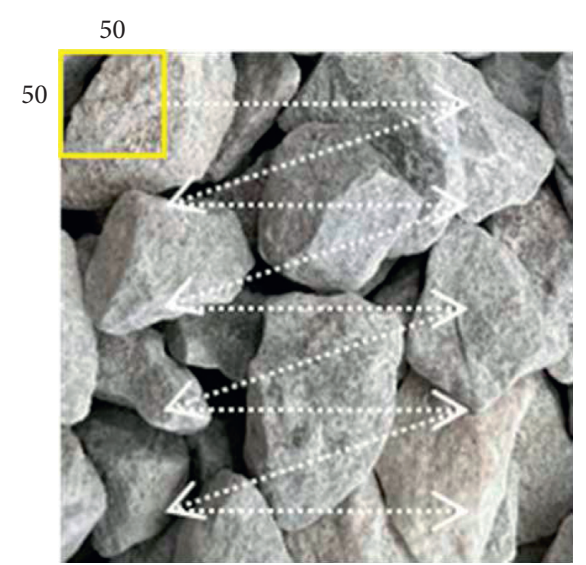

(a)

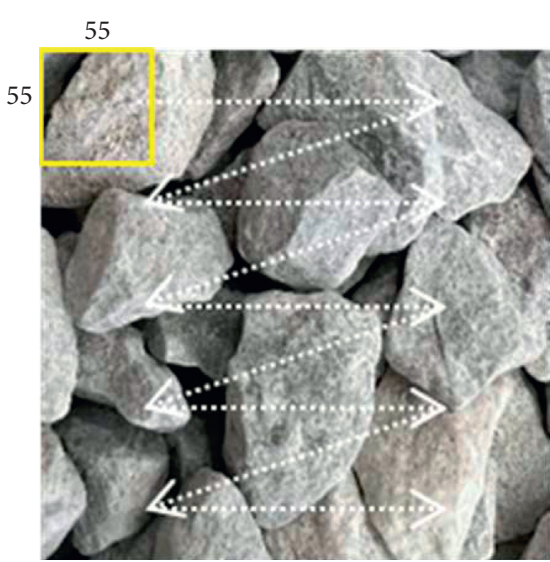

(b)

Figure 8: (a) The minimum sliding window; (b) the increment is 5 pixels.

same particle, resulting in multiple bounding boxes. In this case, we take the average value as a new single window, as shown in Figure 9.

\section{Quantitative Shape Analysis}

3.1. Calculation of Length Flatness of Particles. Common first-scale morphological indicators of particles include flatness, form factor, sphericity, and others. They are mostly used to measure the similarity between the overall contour of particles and the contour of the standard graph of the same diameter (round, square particles, etc.). Also, they are the indicators that have the greatest influence on the overall morphology and mechanical behavior of particles. Among them, the length flatness is widely used in engineering because of its simple measurement and simple concept. Although the existing definitions of length flatness indexes are very diversified, some basic geometric properties of particles are often adopted by most indexes and become common factors of the index system (as shown in Table 1). Therefore, the calculation of these basic indexes is the premise of the calculation of the length flatness index.

Feret's diameter Fer $(a)$ of a particle describes the vernier caliper length of a particle at a certain direction angle, which is the basis of the index of particle length flatness. For the directional angle $a_{i}$ of a particular particle $O_{p 1}$, the Feret diameter Fer $\left(a_{i}\right)$ corresponding to it can be given by the following equation:

$$
\begin{gathered}
O_{p 1}^{\prime}=\left(\begin{array}{cc}
i_{1}^{\prime} & j_{1}^{\prime} \\
i_{2}^{\prime} & j_{2}^{\prime} \\
i_{3}^{\prime} & j_{3}^{\prime} \\
\ldots & \cdots \\
i_{k}^{\prime} & j_{k}^{\prime}
\end{array}\right)=\left(\begin{array}{cc}
i_{1} & j_{1} \\
i_{2} & j_{2} \\
i_{3} & j_{3} \\
\ldots & \ldots \\
i_{k} & j_{k}
\end{array}\right)\left(\begin{array}{cc}
\cos \left(-a_{i}\right) & \sin \left(-a_{i}\right) \\
-\sin \left(-a_{i}\right) & \cos \left(-a_{i}\right)
\end{array}\right) \\
\operatorname{Fer}\left(a_{i}\right)=\max \left(i_{1}^{\prime}, i_{2}^{\prime} \ldots i_{k}^{\prime}\right)-\min \left(i_{1}^{\prime}, i_{2}^{\prime} \ldots i_{k}^{\prime}\right)
\end{gathered}
$$

where the function of equation (1) is to rotate the particle so that the direction corresponding to $a_{i}$ is parallel to the $X$ axis.

The corresponding Feret diameter can be easily obtained with known direction angle $a_{i}$ using equation (2). Therefore, when calculating the maximum (minimum) Feret diameter of particles, the most important problem is to find their corresponding direction angle $a_{i}$. The rotation method [18] is commonly used to solve this problem in existing research studies. The essence of the rotation method is to enumerate the direction angles between 0 and $180^{\circ}$ with a certain angle step length and obtain the approximate maximum (minimum) Feret diameter through comparison by calculating the Feret diameter corresponding to each direction angle. This process is represented geometrically by rotating at angular intervals and measuring the particle profile. In general, the rotation method is simple and intuitive, with high calculation accuracy when the rotation step size is small. It is not affected by the concave part of particles. Therefore, the rotation method can well accomplish the calculation of the particle Feret diameter. Since the rotation method has a certain error when the rotation step is large, the calculation time is relatively great.

The calculation of the length flatness of particles can be divided into two basic ideas: the ratio of direct use of maximum $\left(S_{\text {major }}\right)$ and minimum $\left(S_{\text {major }}\right)$ Feret diameter and the aspect ratio of particle envelopment box. Among them, the definition of particle envelopment box is still controversial in academic circles. A more widely used definition is to take $S_{\text {major }}$ as the length of the enclosing box and define length flatness $E I_{1}=S_{\text {minor }}^{90} / S_{\text {major }}$. However, this method will give incorrect results when calculating the particles of the rectangle (or approximately rectangle). The other definition builds the bounding box indicator $E I_{2}=S_{\text {minor }} / S_{\text {minor }}^{90}$ based on $S_{\text {major. }}$. This method tends to get a smaller bounding box, and it cannot obtain the correct results when calculating rhombic particles. In fact, since $E I_{1}$ and $E I_{2}$ contain $S_{\text {major }}$ and $S_{\text {major }}$, they are also statistically strongly related to another commonly used metric, $E I_{3}=S_{\text {minor }} / S_{\text {minor }}$. 


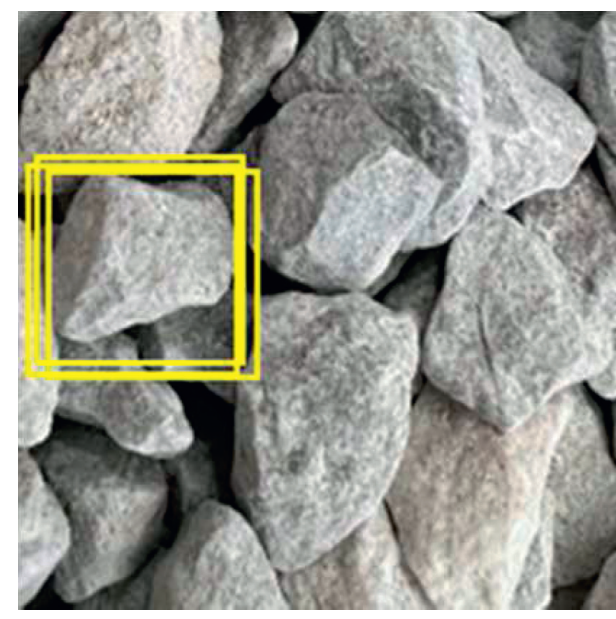

(a)

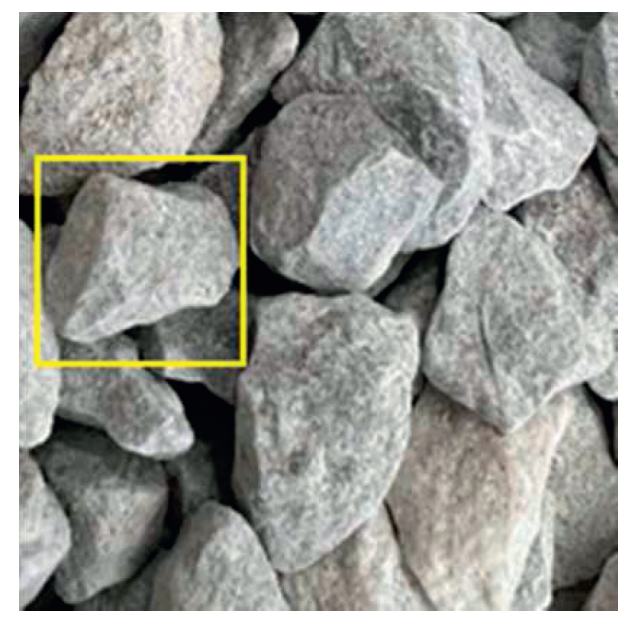

(b)

FiguRE 9: (a) Several bounding box of a particle; (b) the average bounding box.

TABLE 1: Basic particle morphology indicators that are widely used.

\begin{tabular}{lcc}
\hline Parameter & Symbol & Geometric meaning \\
\hline Particle size & $\operatorname{Ar}$ & The area bounded by a particle contour \\
Particle circumference & $\operatorname{Per}$ & Particle contour perimeter \\
Feret's diameter & Fer $(a)$ & "Ruler length" of corresponding direction angle $a$ \\
Length scale & $S_{\text {major }}$ & Maximum Feret's diameter \\
Vertical scale & $S_{\text {major }}^{90} S_{\text {major }}^{90}$ & Feret's diameter orthogonal to the direction corresponding to the long scale (short scale) \\
Short scale & $S_{\text {minor }}$ & Minimum Feret's diameter \\
\hline
\end{tabular}

In this study, referring to existing studies [19-21], $E I_{3}$ is selected as the index to evaluate the length and flatness of particles. A total of 991 real images of subgrade rockfill particles were extracted, and the calculation results of $\mathrm{EI}_{3}$ can be obtained, as shown in Figure 10. From the figure, it can be seen that the results of $E I_{3}$ approximately follow a normal distribution, with a mean value of 0.73 and a width from 0.4 to 1.2 .

3.2. Calculation of Edge Angles and Roundness of Particles. Barrett [22] expounded the advantages of the independence of grain morphology indexes among scales. However, in the existing research, the edge angle index is often difficult to maintain its independence from the first- and third-scale indexes. For example, $R I$ calculation formula contains the basic indexes of the first scale, such as particle circumference and area, which are highly dependent on the morphological characteristics of the first scale. The AT index cannot guarantee its independence from the third-scale morphology because the grain angular and grain roughness effects are considered simultaneously. To solve this problem, Liu et al. [23] proposed an edge angle index $A I$ based on a convex hull. The index is defined as the ratio of the equivalent elliptic perimeter of the particle to the circumference of the convex hull of the particle, where the equivalent ellipse of a particle is an ellipse with the same length flatness and area as the particle, and its semimajor axis $(a)$ and semiminor axis $(b)$ are given by the following equation:

$$
\begin{aligned}
& a=\sqrt{\frac{\mathrm{Ar}}{\pi E I_{3}}}, \\
& b=a E I_{3} .
\end{aligned}
$$

$A I$ indicator has the following characteristics:

(1) For smooth particles, their circumference should be close to its equivalent ellipse circumference. The more trenchant the edges and corners particles are, the longer the perimeter will be, leading to $A I$ value declining. Therefore, $A I$ indicator can be used to reflect the degree of angular.

(2) By convex hull, the influence of particle contour roughness is excluded and the independence with the third-scale index is maintained.

(3) By comparing the equivalent ellipse with the same length flatness, the influence of length flatness is normalized and the dependence with the first-scale index is reduced.

The characteristics (2) and (3) guarantee the independence of this index, which is an advantage compared with other indexes. However, when the convex hull is used to exclude the roughness of the contour, the angular fluctuation of the particle will also be excluded, so that the concave part of the particle cannot be considered in this index. Therefore, this index has been modified in this study. Firstly, B-spline resampling technique was used to process the 


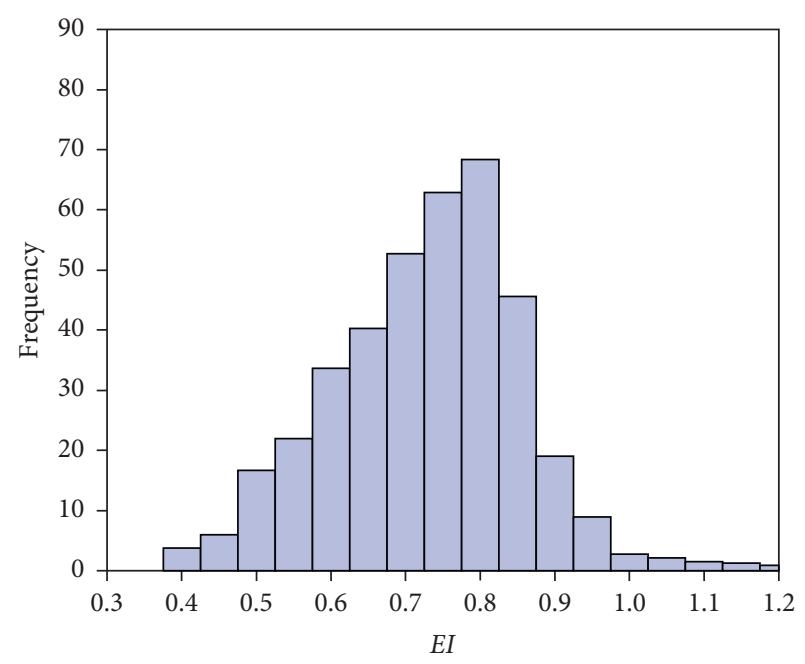

Figure 10: Statistical results of the length flatness index calculated by the rotation method.

original particle outline, eliminating particle roughness and retaining edges and corners. Then, the improved edge angle $A I 2$ is obtained by directly comparing the smoothed particle circumference $\operatorname{per}_{s}$ with the equivalent ellipse circumference $\mathrm{Per}_{e}$ :

$$
A I_{2}=\frac{\mathrm{Per}_{e}}{\mathrm{Per}_{s}}
$$

A total of 991 real subgrade rockfill particles is used to calculate the contour edge angle values, and the statistical curve is drawn in Figure 11. It can be seen that the edge angle approximately follows a negative skewness distribution.

3.3. Calculation of Particle Contour Roughness. Particle roughness describes the degree of fine fluctuation of the particle surface. In this section, the conventional particle roughness based on polar radius is improved. By using $\mathrm{B}$-spline resampling to calculate the particle smooth and normal vector, an index for quantitative analysis of particle contour roughness was proposed. Based on the above threesegment intersection determination algorithm, a roughness index with scale invariance is proposed in this study. The calculation is as follows:

(1) B-spline resampling is carried out for the particle contour, and the normal vector of each sampling point is calculated

(2) A line segment is made at the sampling point along the normal vector, so that its length is equal to the radius $R_{\text {eq }}$ of the equal-area circle (to ensure that the line segment has an intersection)

(3) The line segment is judged to be intersecting with the original particle contour, and the distance dist ${ }_{i}$ from the nearest intersection point to the sampling point is calculated

(4) Repeat steps (2) and (3) for all sampling points

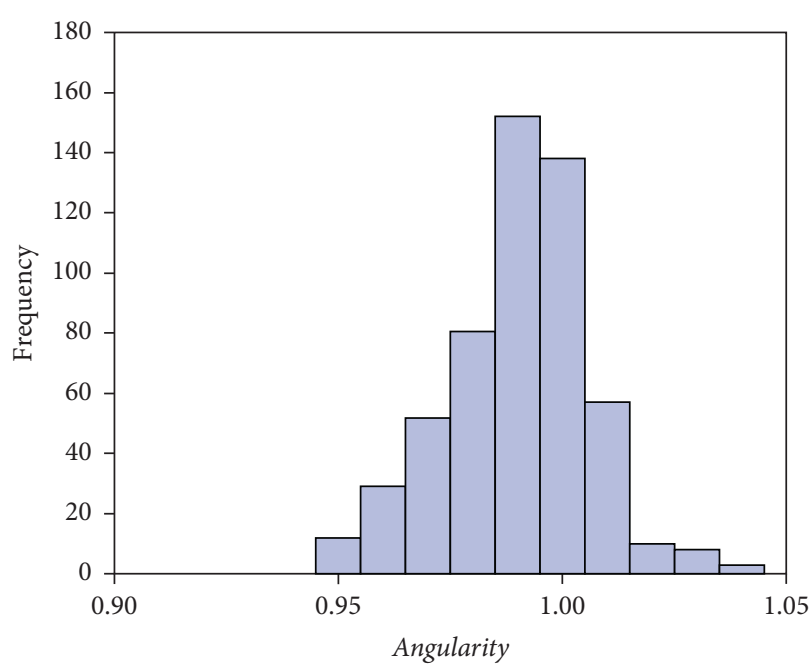

FIGURE 11: Statistical results of edge angles and improved posterior edge angles.

Based on the above steps, roughness index $R_{g}$ can be obtained as follows:

$$
R_{g}=\sum_{i=1}^{n} \frac{\text { dist }_{i}}{n R_{\mathrm{eq}}}
$$

where $R_{\text {eq }}$ ensures the scale invariability of the index; that is, $R_{g}$ remains unchanged when the particle contour is equilateral magnified.

The angle values of edges of 991 real subgrade rockfill particle contours are calculated, and the statistical curves are plotted in Figure 12, which is approximately a positive skewness distribution.

\section{Application to DEM Simulation}

4.1. Simulation of Earth Compaction of Rockfill Subgrade. Affected by the actual construction scale of the subgrade project, particle gradation and the irregular shape particles are adopted in the simulation, and the adoption of the 3D model will greatly increase the workload of the computer. Therefore, the two-dimensional model can also be used to simulate and analyze the particle motion characteristics of rockfill in the compaction process from a microscopic perspective.

In this study, discrete element software PFC2D was used to simulate the compaction process of rockfill in two dimensions. To ensure that the roller vibrator rolls rather than sliding during the movement, the relationship between the angular velocity $w$ and the horizontal velocity $v$ of the vibrator is shown in the following equation:

$$
v=w \times R,
$$

where, $v, w$, and $R$ are the horizontal velocity, angular velocity, and radius of the vibration wheel, respectively.

The simulated material is rockfill for subgrade construction, and the linear elastic contact model is adopted in the discrete element model, which is widely used in similar studies [24]. The mesoscopic parameters used in the 


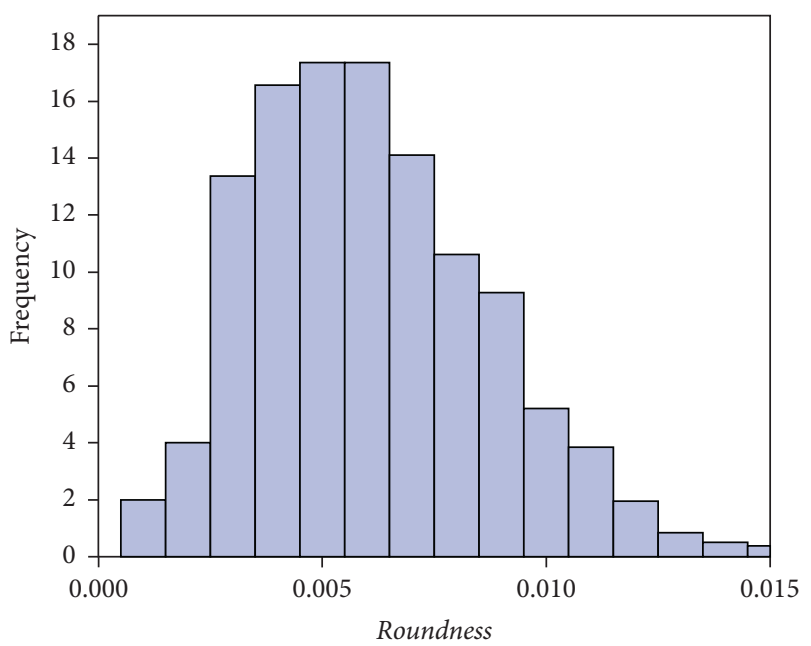

Figure 12: Statistical results of roughness index.

simulation refer to the research results of Liu et al. [24], and the specific parameters are shown in Table 2.

Referring to the research of Goldenberg and Goldhirsch [25], Ma et al. [11], Zhou et al. [9], Alaei and Mahboubi [26] and Nie et al. [12], in this study, 5 groups of two-dimensional particles with different edge angles were generated, with 8 samples in each group, as shown in Figure 13. To exclude the influence of elongation on the simulation results, the elongation of particles was controlled between 0.97 and 1.00 . Note that particles of roundness 1 are standard circles and are not shown in Figure 13. In addition, the effect of particle roughness was not considered in this study, that is, $D_{8}=0.0$.

In PFC2D, the clump function can realize the fitting of complex particle contour, with the fitting accuracy control parameters distance (range 0-180) and ratio (range 0.0-1.0). The larger the distance, the smaller the ratio, and the fitted results are closer to the real model. However, more computational memory and running time are consumed. In this study, the distance was set as 162 , and the ratio was set as 0.25 . Figure 14 shows a clump model filled and generated under this setting. It can be seen that the set of two parameters is sufficient to reflect the external contour of the particle.

Coarse particle packing with a diameter greater than $10 \mathrm{~mm}$ was used in this simulation to improve the calculation efficiency, and the distribution of the size of the packing was calculated. The results show that the content percentage of diameter ranging from 10 to $20 \mathrm{~mm}, 20$ to $30 \mathrm{~mm}, 30$ to $40 \mathrm{~mm}, 40$ to $50 \mathrm{~mm}$, and 50 to $60 \mathrm{~mm}$ is $17 \%$, $20 \%, 25 \%, 20 \%$, and $18 \%$, respectively.

The packing model was set in the range of height $(H) \times$ width $(W)=0.8 \mathrm{~m} \times 8 \mathrm{~m}$. The initial model is generated by gravity sedimentation. The process is as follows:

(1) In the $2 H \times W$ range, contactless particles slightly larger than the total volume of the target were randomly generated according to the particle size range and content, as shown in Figure 15(a).

(2) Under the action of gravity, particles fall freely, when the particle system reaches a quasi-static state and stops running, as shown in Figure 15(b).

(3) In order to ensure that fillers with different roundnesses are compacted at the same thickness, redundant particles above the thickness line are removed to simulate the leveling process. The final leveling filler is shown in Figure 15(c).

(4) A rigid body circular element is generated to simulate the roller. The movement parameters and attribute parameters of the roller are determined in Tables 2 and 3. The chord wave vibration load is applied to the filler during the movement of the roller, as shown in Figure 15(d). And the simulation is stopped after repeated rolling 12 times.

4.2. The Macroresult. Figure 16(a) shows the porosity of fillers with different roundnesses varying with the number of rolls. Obviously, with the increase in the number of rolling times, the pores first decreased sharply and then remained unchanged. But the correlation between roundness and vibratory compaction was not strong. The initial porosity was extracted (shown in Figure 16(b)), and it was found that with the increase of roundness, the initial porosity first decreased and then increased, which was consistent with the previous research results related to particle roundness [27]. Figure $16(\mathrm{c})$ shows the normalized porosity rate $\Delta n$ obtained after conversion using equation (7). It is found that the small roundness of the particle indicates irregular shape and great drop in the particle porosity compared to the initial state. The normalized porosity rate is obtained as

$$
\Delta n=\frac{n_{0}-n_{i}}{n_{0}} \times 100 \%
$$

where $n_{0}$ and $n_{i}$ represent the porosity corresponding to the initial porosity and the porosity corresponding to the $i$ th pass after rolling, respectively.

Figure 17 shows the cumulative settlement amount of the pavement corresponding to each roll pass of the filler, which shows a trend similar to the normalized pore change rate. It shows a trend of rapid settlement in the early stage and a trend of slow settlement in the later stage. This trend is consistent with the actual change in the monitored value in construction [24]. The indirect results showed that the particles sank under the vibratory compaction condition, which made the fillers squeeze and approach each other and rearrange the particles. This results in a smaller porosity, showing the changing trend as shown in Figure 16(a). In addition, a small value of roundness of the particles represents the great settlement of the fillers. Therefore, in 
TABLE 2: Microscale parameters used in the DEM simulations.

\begin{tabular}{lcccccc}
\hline $\begin{array}{l}\text { Microscopic } \\
\text { parameters }\end{array}$ & $\begin{array}{c}\text { The normal } \\
\text { stiffness, } k n(\mathrm{~N} / \mathrm{m})\end{array}$ & $\begin{array}{c}\text { The tangential } \\
\text { stiffness, } k s(\mathrm{~N} / \mathrm{m})\end{array}$ & $\begin{array}{c}\text { The coefficient of } \\
\text { friction, fric }\end{array}$ & $\begin{array}{c}\text { Local } \\
\text { damp }\end{array}$ & $\begin{array}{c}\text { Normal/tangential } \\
\text { damping } d p_{n}, d p_{t}\end{array}$ & $\begin{array}{c}\text { Density }\left(\mathrm{kg} / \mathrm{m}^{3}\right) \\
\text { (a) }\end{array}$ \\
\hline Value (particle) & $1.5 \times 10^{7}$ & $2.9 \times 10^{7}$ & 0.5 & 0.9 & 0.5 & 2600 \\
Value (wheel) & $1.0 \times 10^{9}$ & $1.0 \times 10^{9}$ & 0.3 & 0.0 & 0.5 & 7800 \\
\hline
\end{tabular}

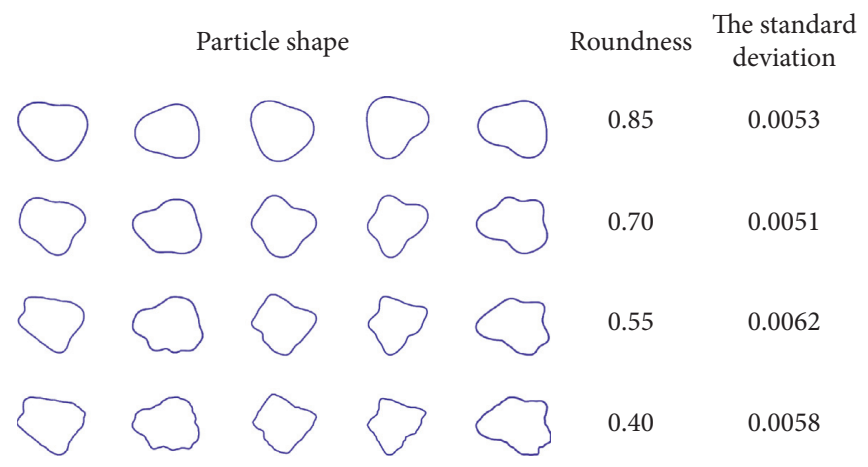

FIgURE 13: Two-dimensional particle models with different roundnesses.

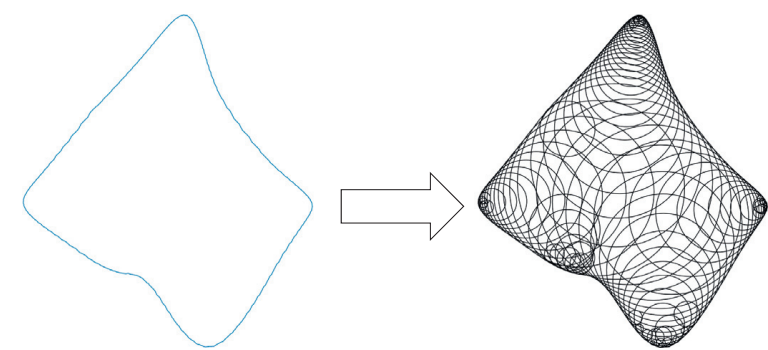

Figure 14: A DEM-simulated virtual particle from a Fourier-based particle contour.

practical engineering, the irregular shape of the packing requires more rolling times.

\subsection{The Micromechanical Results}

4.3.1. Coordination Number. Coordination number is an important index to characterize the internal structure of particle systems. For the irregular particle model composed of Clump, there are two different definitions of coordination number: one is the number of particles in contact with the specified particles, called the coordination number of particles; and the other is the number of contacts belonging to a given particle, called the contact coordination number.

Thornton [28] pointed out that there are particles in granular materials that do not contact with other particles or only contact with one particle, and such particles have no contribution to the overall stability of the material. Therefore, the mechanical average particle coordination number is defined as follows:

$$
C N=\frac{2 C-N_{1}}{N-N_{0}-N_{1}},
$$

where $C$ is the total number of particle contact, $N$ is the total number of particles, and $N_{0}$ and $N_{1}$ are the numbers of particles in contact with zero or one particle in the particle system. Figure 18 shows the relationship between the mechanical particle coordination number and the rolling pass number of fillers with different roundnesses. For each roundness case, the particle coordination number showed a slight upward trend as the number of rolling passes increased; that is, the number of particles in contact with a certain particle increased. This was related to the overall looseness of the packing and reflected that the packing compactness increased as the number of rolling passes increased. For a certain roundness filler, the increase in the coordination number will lead to the enhancement of interlock between the particles, thus improving the antirotation ability of the particles. In addition, large roundness values of particles represent a large coordination number of the particles, indicating that the rounder particles are more likely to contact multiple particles.

Figure 19 shows the relationship between average contact coordination number and rolling pass number of fillers with different roundnesses. Similar to the variation trend of the particle coordination number, the contact coordination number of a certainly shaped particle increased only slightly on the whole. However, for the particles with different roundnesses, smaller roundness corresponds to a larger contact coordination number of the particles, indicating that the interlock phenomenon is more obvious. 


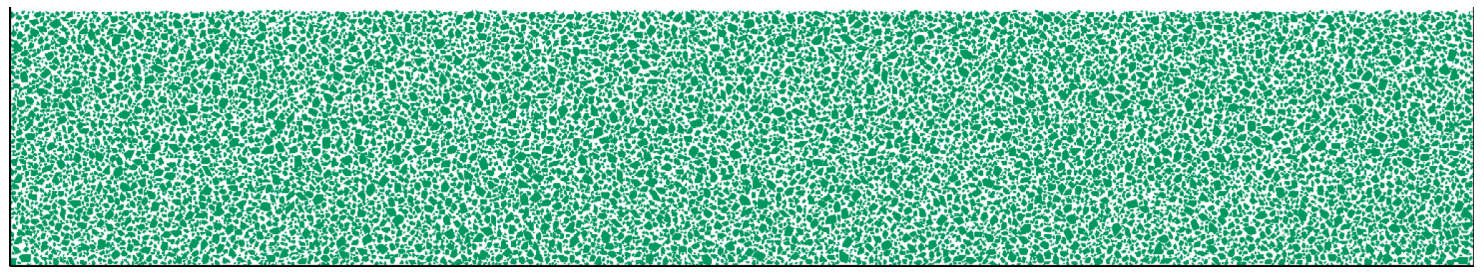

(a)

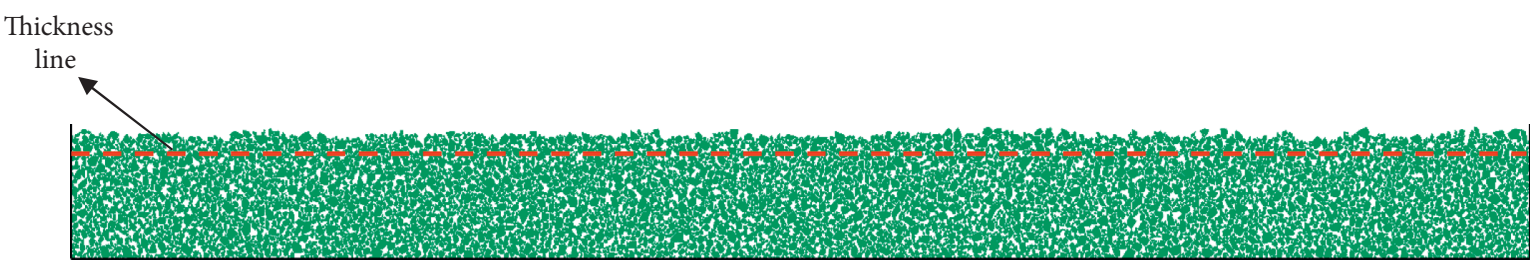

(b)

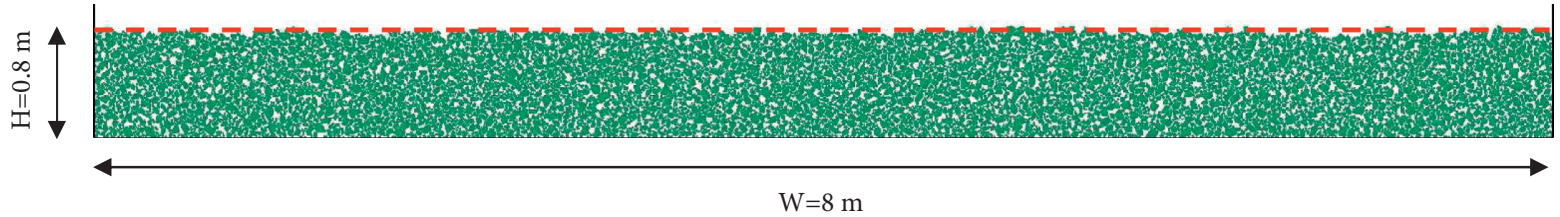

(c)
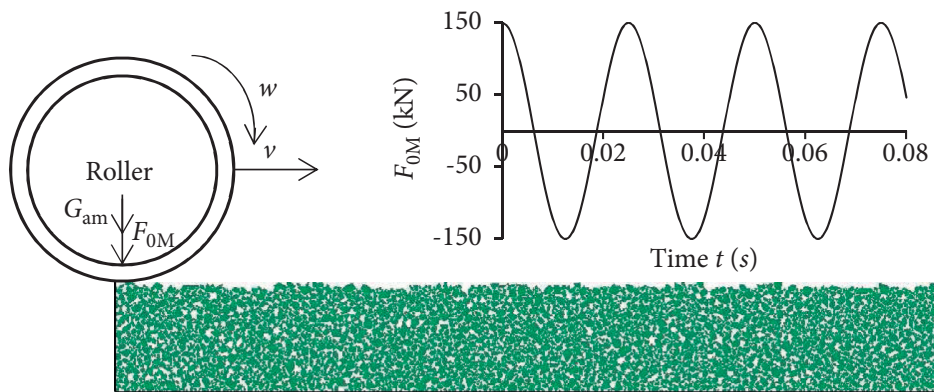

Time $t(s)$

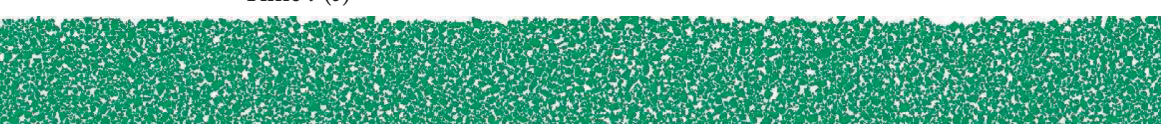

(d)

FIGURE 15: PFC simulation of vibratory compaction, including dumping, spreading, and compaction. (a) The contactless model; (b) sedimentation accumulation; (c) leveling; (d) vibratory compaction.

TABLE 3: External load parameters.

\begin{tabular}{lcccccc}
\hline $\begin{array}{l}\text { Rolling wheel } \\
\text { parameters }\end{array}$ & $\begin{array}{c}\text { Quality of } \\
\text { grinding wheel, } G \\
(\mathrm{~kg})\end{array}$ & $\begin{array}{c}\text { Grinding wheel } \\
\text { diameter, } D(\mathrm{~mm})\end{array}$ & $\begin{array}{c}\text { Grinding wheel } \\
\text { width, } b(\mathrm{~mm})\end{array}$ & $\begin{array}{c}\text { Vibration } \\
\text { frequency, } f(\mathrm{~Hz})\end{array}$ & $\begin{array}{c}\text { Exciting force, } F_{0}(\mathrm{kN}) \\
(\mathrm{m} / \mathrm{s})\end{array}$ & $\begin{array}{c}\text { Speed, } v \\
\text { Rolling pass }\end{array}$ \\
$\begin{array}{l}\text { The actual } \\
\text { parameter }\end{array}$ & $1 \times 10^{4}$ & 1500 & 2000 & 40 & 300 & 1 \\
$\begin{array}{l}\text { Simulation } \\
\text { parameters }\end{array}$ & $5 \times 10^{3}$ & 1500 & 1000 & 40 & 150 & 1 \\
\hline
\end{tabular}

4.3.2. Particle Rotation Momentum. Rotation is an important relative motion between particles in contact. The rotation between particles is related to the rotation behavior of individual particles along the reference axis. Figures 20(a) and 20(b) show the cumulative rotational momentum of fillers with different roundnesses along with clockwise and counterclockwise directions as a result of rolling. It can be seen from Figure 20 that the rotation momentum of the packing in the clockwise and counterclockwise directions is basically the same. Under a certain roundness, the rotation value of the particles increases with the increase in the number of rolling times, and the increase tends to slow down. This is because the increase in the particle coordination number and contact coordination number enhances the interlock between particles and hinders the rotation of particles. In addition, the smaller roundness of the particle represents the smaller rotational momentum of the particle, indicating that the more irregular the particle is, the stronger the antirotation ability is. The presumed reason is that the 


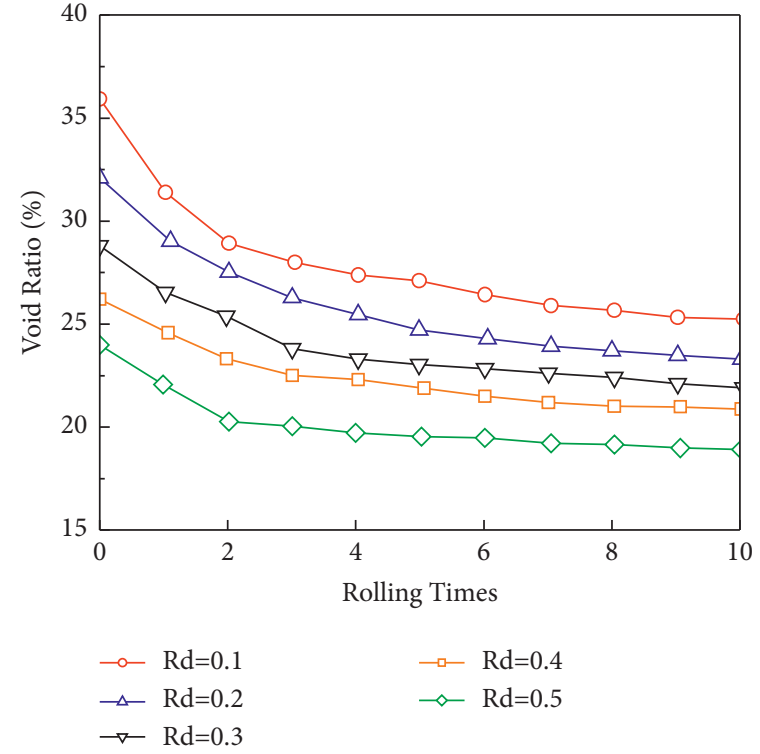

(a)
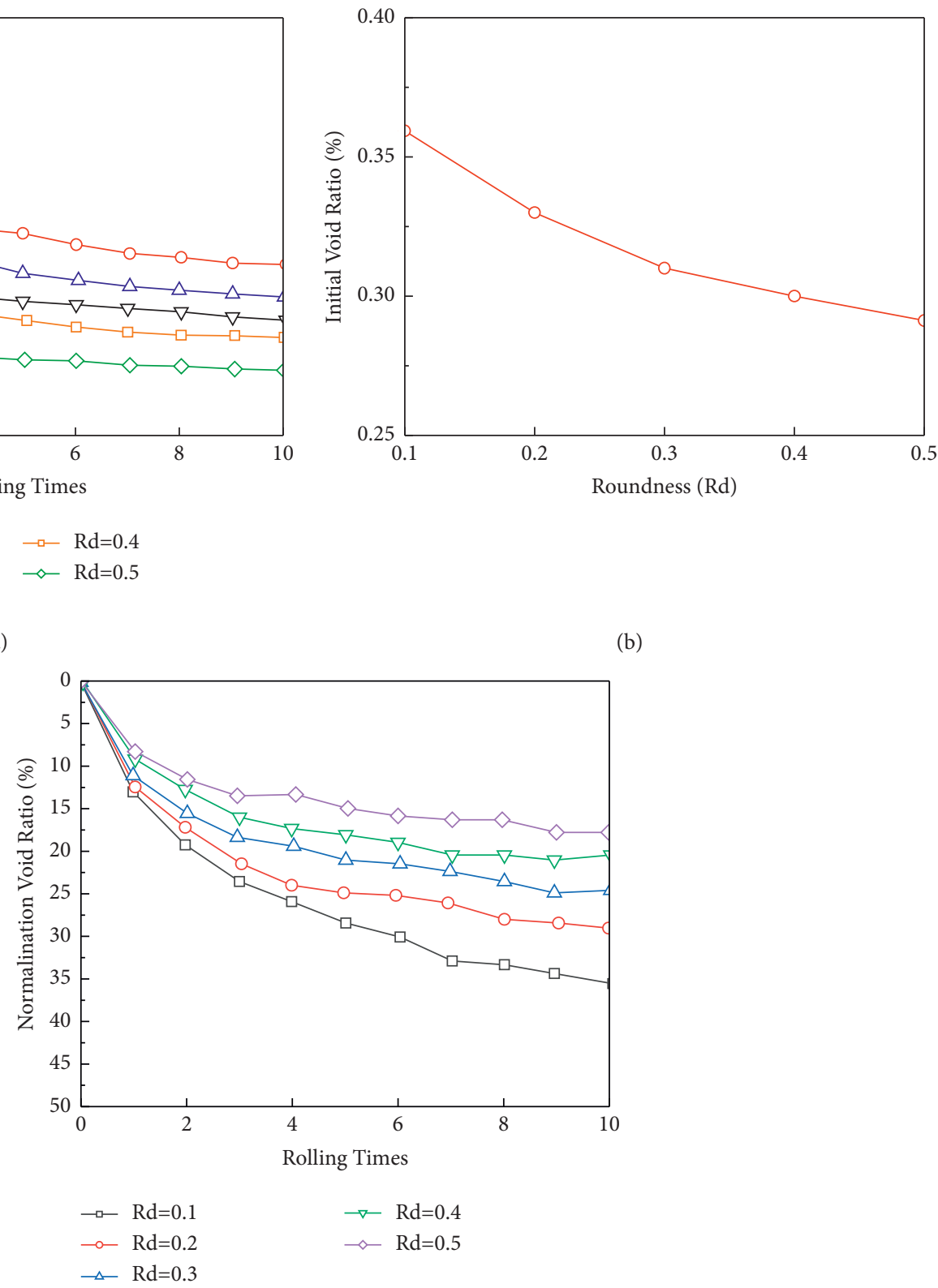

(c)

Figure 16: The relationship between the compactor pass and pore change of fillings with different roundnesses. (a) Porosity; (b) initial porosity; (c) normalized void change rate.

particles with lower roundness have a larger contact coordination number, which increases the interlock effect between the particles and thus hinders the rotation of the particles.

Figure 21(b) shows the vertical displacement of the packing. It showed a similar trend to the cumulative settlement; that is, with the increase in the number of rolling times, the particles gradually sank and the downward trend gradually slowed down. In addition, for fillers with different roundnesses, the smaller the particle roundness is, the greater the vertical displacement is. Estrada et al. [29] pointed out that when the particles in contact have relative movement, irregular particles with stronger antirotation ability will be forced to slide along the contact surface, so as to regulate the overall deformation of the particle system. Therefore, more irregular particles are more prone to relative sliding between particles, resulting in larger vertical displacement of particles. 


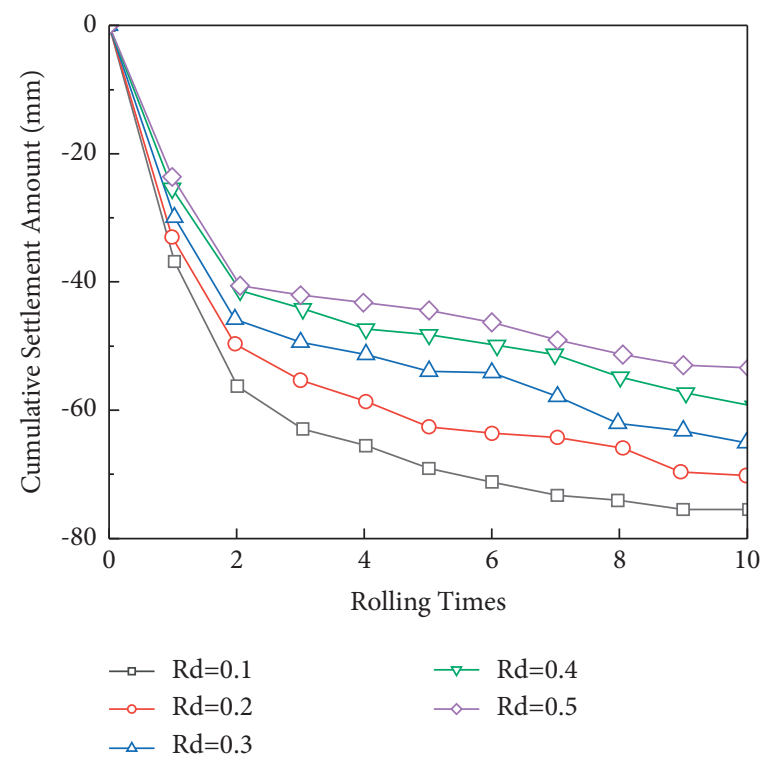

FIGURE 17: Cumulative settlement-compactor pass responses for fillings with different roundnesses.

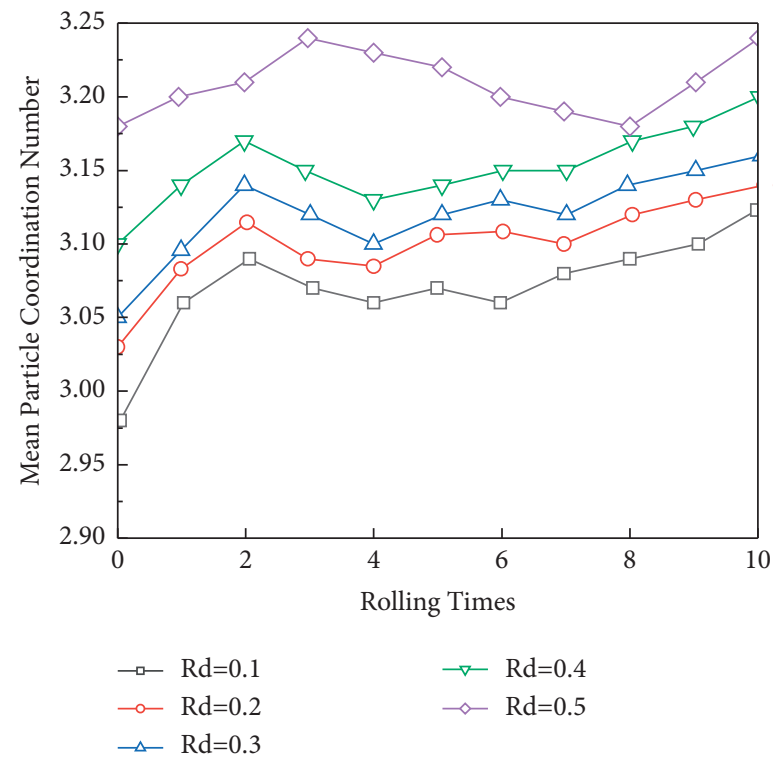

FIGURE 18: Mean particle coordination number-compactor pass responses for fillings with different roundnesses. 


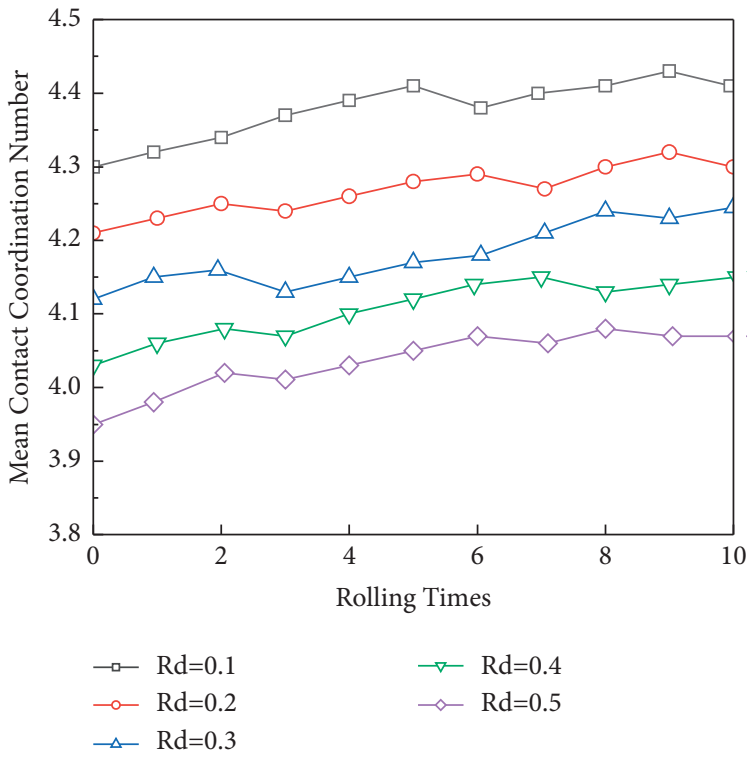

Figure 19: Mean contact coordination number-compactor pass responses for fillings with different roundnesses.

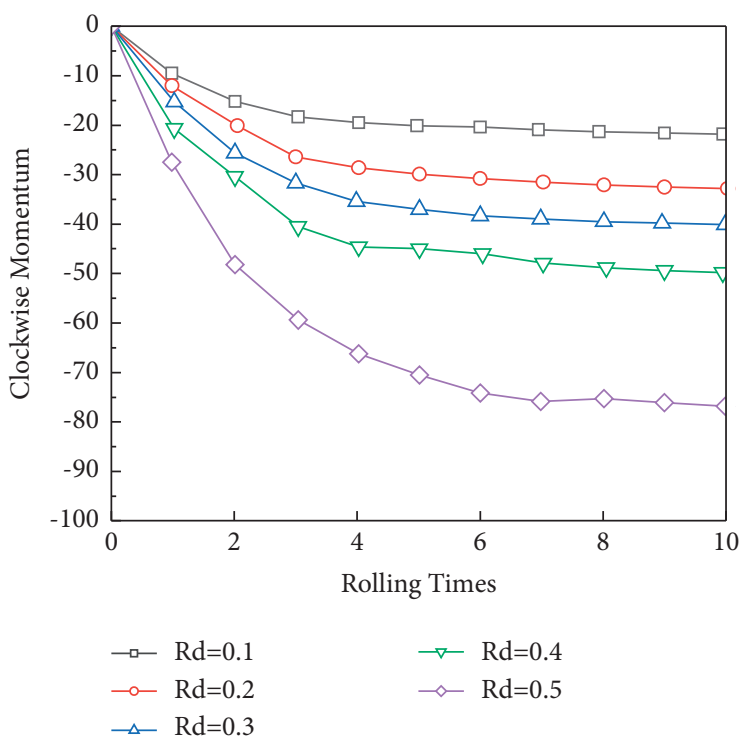

(a)

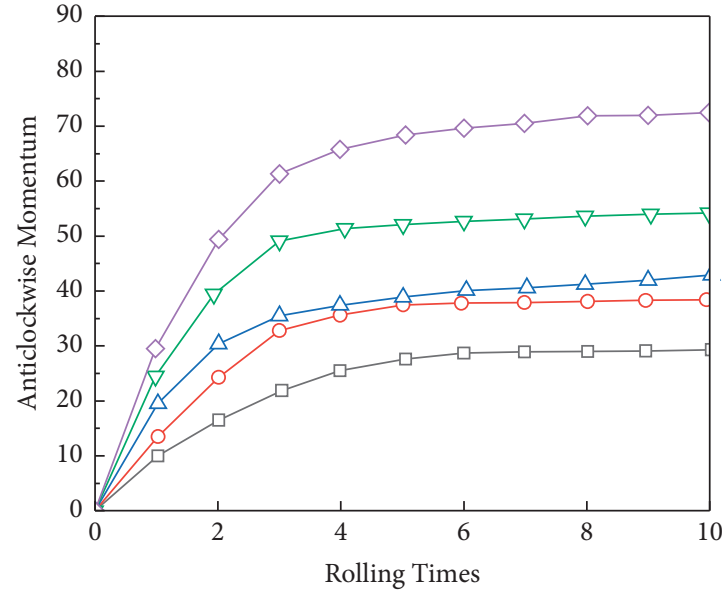

$$
\begin{aligned}
& \rightarrow-\mathrm{Rd}=0.1 \\
& \rightarrow-\mathrm{Rd}=0.4 \\
& \leadsto-\mathrm{Rd}=0.5
\end{aligned}
$$$$
\text { (1) }
$$

(b)

FIGURE 20: Particle rotation magnitude-compactor pass responses for fillings with different roundnesses. (a) Clockwise momentum; (b) rotational momentum in reverse time. 


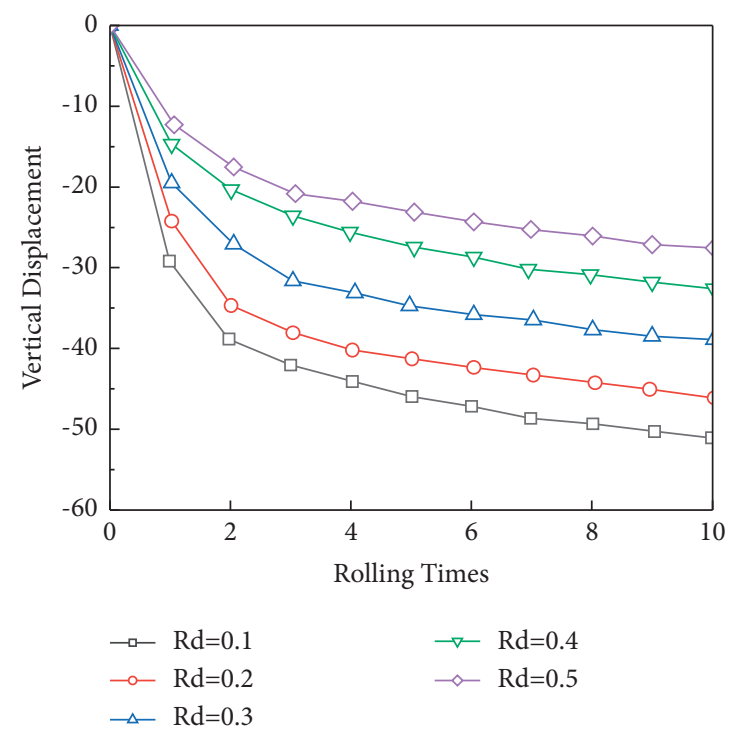

FIgURE 21: Displacement components of fillings in the vibration rolling process: vertical displacement.

\section{Conclusion}

Rockfill material is of great significance in the subgrade construction industry. The mechanical properties can be affected by the irregularity and variability of particle shape , which can be explored by discrete element simulation. In this study, the subgrade rockfill particle outlines are firstly extracted based on machine learning algorithms, including AdaBoost, Cascade, and sliding windows. Then, the shape evaluation indexes are quantified and the statistical analysis of each index is presented. In addition, the DEM simulation is implemented on the compaction of rockfill subgrade. Based on the simulation, the macroanalysis on the void ratio and cumulative settlement is given. Finally, the microanalysis on the particle coordination number, rotation momentum, and displacement is studied. The specific results are as follows:

(1) 1206 pictures of the subgrade rockfill particles are taken as the training data. By training a strong classifier through AdaBoost in each stage of Cascade, only a small amount of pictures can pass all $s$ stages in the Cascade algorithm. These pictures contain a complete outline of particles. Then, using sliding window and adding boundaries of particles, the final window of a particle can be obtained indicating that the particle is well extracted.

(2) The quantitative shape analysis is presented. For length flatness, 991 real particles show an approximately normal distribution, whereas the edge angle and roughness show a negative skewness distribution and positive skewness distribution, respectively.

(3) With the increase in the number of rolling passes, the porosity of the packing decreases gradually and the settlement increases gradually, and the change rate is fast at the beginning and slow in the end. In addition, smaller roundness of the particles represents a more obvious porosity drop and the greater settlement of the filler compared with the initial state.

(4) Under the rolling load, the particle coordination number and contact coordination number of the filler increased slightly. The smaller the roundness is, the larger the contact coordination number is, which increases the interlock effect and hinders the rotation of the particles. The horizontal displacement of particles shows a certain fluctuation, whereas the vertical displacement increases gradually. In addition, the smaller the roundness is, the larger the vertical displacement is and the smaller the horizontal displacement is.

\section{Data Availability}

The data used in the article are included in the article.

\section{Conflicts of Interest}

The authors declare that they have no conflicts of interest.

\section{References}

[1] A. Varadarajan, K. G. Sharma, K. Venkatachalam, and A. K. Gupta, "Testing and modeling two rockfill materials," Journal of Geotechnical and Geoenvironmental Engineering, vol. 129, no. 3, pp. 206-218, 2003.

[2] J. Zhang, J. Li, Y. Yao, J. Zheng, and F. Gu, "Geometric anisotropy modeling and shear behavior evaluation of graded crushed rocks," Construction and Building Materials, vol. 183, pp. 346-355, 2018.

[3] R. Pang, B. Xu, X. Kong, D. Zou, and Y. Zhou, "Seismic reliability assessment of earth-rockfill dam slopes considering strain-softening of rockfill based on generalized probability density evolution method," Soil Dynamics and Earthquake 
Engineering, vol. 107, pp. 96-107, 2018.

[4] R. Deluzarche and B. Cambou, "Discrete numerical modelling of rockfill dams," International Journal for Numerical and Analytical Methods in Geomechanics, vol. 30, no. 11, pp. 1075-1096, 2006.

[5] J. Li, J. Zheng, Y. Yao, J. Zhang, and J. Peng, "Numerical method of flexible pavement considering moisture and stress sensitivity of subgrade soils," Advances in Civil Engineering, vol. 2019, Article ID 7091210, 10 pages, 2019.

[6] W. Hui, J. Li, F. Wang, J. Zheng, Y. Tao, and Y. Zhang, "Numerical investigation on fracture evolution of asphalt mixture compared with acoustic emission," International Journal of Pavement Engineering, vol. 2021, pp. 1-10, 2021.

[7] J. Raisianzadeh, S. Mohammadi, and A. A. Mirghasemi, "Micromechanical study of particle breakage in $2 \mathrm{~d}$ angular rockfill media using combined dem and xfem," Granular Matter, vol. 21, no. 3, p. 48, 2019.

[8] W. Song, F. Xu, H. Wu, and Z. Xu, "Investigating the skeleton behaviors of open-graded friction course using discrete element method," Powder Technology, vol. 385, pp. 528-536, 2021.

[9] W. Zhou, G. Ma, X. Chang, and C. Zhou, "Influence of particle shape on behavior of rockfill using a three-dimensional deformable dem," Journal of Engineering Mechanics, vol. 139, no. 12, pp. 1868-1873, 2013.

[10] J. Li, J. Zhang, G. Qian, J. Zheng, and Y. Zhang, "Three-dimensional simulation of aggregate and asphalt mixture using parameterized shape and size gradation," Journal of Materials in Civil Engineering, vol. 31, no. 3, Article ID 04019004, 2019.

[11] G. Ma, W. Zhou, X.-L. Chang, and W. Yuan, "Combined fem/ dem modeling of triaxial compression tests for rockfills with polyhedral particles," International Journal of Geomechanics, vol. 14, no. 4, Article ID 04014014, 2014.

[12] Z. Nie, Y. Zhu, X. Wang, and J. Gong, "Investigating the effects of Fourier-based particle shape on the shear behaviors of rockfill material via DEM," Granular Matter, vol. 21, no. 2, p. 22, 2019.

[13] M. Jones and P. Viola, "Fast multi-view face detection," Mitsubishi Electric Research Lab TR-20003-96,vol. 3, no. 14, p. 2, 2003.

[14] R. Lienhart and J. Maydt, "An extended set of haar-like features for rapid object detection," in Proceedings of the International Conference on Image Processing, vol. 1, September 2002.

[15] J. Zheng and R. D. Hryciw, "Identification and characterization of particle shapes from images of sand assemblies using pattern recognition," Journal of Computing in Civil Engineering, vol. 32, no. 3, Article ID 04018016, 2018.

[16] P. Viola and M. Jones, "Fast and robust classification using asymmetric adaboost and a detector cascade," Advances in Neural Information Processing Systems, vol. 14, pp. 1311-1318, 2001.

[17] A. Dosovitskiy, J. T. Springenberg, M. Riedmiller, and T. Brox, "Discriminative unsupervised feature learning with convolutional neural networks," Advances in Neural Information Processing Systems, vol. 1, pp. 766-774, 2014.

[18] J. Cardona, C. Ferreira, J. McGinty et al., "Image analysis framework with focus evaluation for in situ characterisation of particle size and shape attributes," Chemical Engineering Science, vol. 191, pp. 208-231, 2018.

[19] Z. Lianheng, H. Dongliang, D. Han-Cheng, Z. Shuaihao, and L. Dejian, "Reconstruction of granular railway ballast based on inverse discrete Fourier transform method," Granular Matter, vol. 19, no. 4, p. 74, 2017.
[20] W. Song, B. Huang, X. Shu, J. Stránský, and H. Wu, "Interaction between railroad ballast and sleeper: a DEM-FEM approach," International Journal of Geomechanics, vol. 19, no. 5, Article ID 04019030, 2019.

[21] H. Wu, L. Zhu, W. Song, Z. Xu, F. Xu, and H. Gong, "Impact performance of ballast by incorporating waste tire-derived aggregates," Construction and Building Materials, vol. 288, Article ID 122992, 2021.

[22] P. J. Barrett, "The shape of rock particles, a critical review," Sedimentology, vol. 27, no. 3, pp. 291-303, 1980.

[23] M. L. Hentschel and N. W. Page, "Selection of descriptors for particle shape characterization," Particle \& Particle Systems Characterization: Measurement and Description of Particle Properties and Behavior in Powders and Other Disperse Systems, vol. 20, no. 1, pp. 25-38, 2003.

[24] D. Liu, L. Sun, H. Ma, and W. Cui, "Process simulation and mesoscopic analysis of rockfill dam compaction using discrete element method," International Journal of Geomechanics, vol. 20, no. 6, Article ID 04020047, 2020.

[25] C. Goldenberg and I. Goldhirsch, "Friction enhances elasticity in granular solids," Nature, vol. 435, no. 7039, pp. 188-191, 2005.

[26] E. Alaei and A. Mahboubi, "A discrete model for simulating shear strength and deformation behaviour of rockfill material, considering the particle breakage phenomenon," Granular Matter, vol. 14, no. 6, pp. 707-717, 2012.

[27] Z. Nie, C. Fang, J. Gong, and Z.-Y. Yin, "Exploring the effect of particle shape caused by erosion on the shear behaviour of granular materials via the DEM," International Journal of Solids and Structures, vol. 202, pp. 1-11, 2020.

[28] C. Thornton, "Numerical simulations of deviatoric shear deformation of granular media," Géotechnique, vol. 50, no. 1, pp. 43-53, 2000.

[29] N. Estrada, E. Azéma, F. Radjai, and A. Taboada, "Identification of rolling resistance as a shape parameter in sheared granular media," Physical review. E, Statistical, nonlinear, and soft matter physics, vol. 84, no. 1 Pt 1, Article ID 011306, 2011. 